\section{Internationaler Preis} für moderne Ernährung 2003

Die Organisation der Schweizer Milchproduzenten SMP, Weststrasse 10, 3000 Bern 6, schreibt für 2003 den von ihr gestifteten Internationalen Preis für moderne Ernährung aus. Der Preis im Betrage von Fr. 25 000.- wird einem Wissenschafter aus den Mitgliedstaaten des Internationalen Milchwirtschaftsverbandes* verliehen.

Thema für 2003: Rolle der Ernährung in der Prävention der Adipositas

Eingabefrist: 31. Dezember 2002

Damen und Herren, welche wissenschaftliche Arbeiten zu den gestellten Themen verfasst haben, sind eingeladen, folgende Unterlagen in 3 Exemplaren einzureichen:

- Curriculum vitae;

- Verzeichnis der Arbeiten;

- Liste der Publikationen;

- Sonderdrucke der fünf wichtigsten Arbeiten über das Preisthema, die im Laufe der letzten Jahre veröffentlicht worden sind.

Die Dokumente müssen in deutscher, französischer oder englischer Sprache abgefasst sein und sind zu richten an den Präsidenten der Jury: Herrn Professor Dr. med. Eric Jéquier, Faculté de médecine, Institut de Physiologie, Rue du Bugnon 7, 1005 Lausanne.

* Mitgliedstaaten sind:

Albanien, Australien, Belgien, Dänemark, Deutschland, Estland, Finnland, Frankreich, Griechenland, Grossbritannien, Indien, Iran, Irland, Island, Israel, Italien, Japan, Kanada, Kenya, Kuwait, Litauen, Lettland, Luxemburg, Neuseeland, Niederlande, Norwegen, Österreich, Pakistan, Polen, Russische Föderation, Schweden, Schweiz, Spanien, Südafrika, Tschechische Republik, Ungarn, USA, Zimbabwe.

\section{Prix International de l'alimentation moderne 2003}

La Fédération des Producteurs Suisses de Lait PSL, Weststrasse 10, 3000 Berne 6, met au concours le Prix International de l'alimentation moderne 2003 qu'elle a institué. Ce prix de 25000 francs suisses sera décerné à un scientifique provenant de l'un des pays membres de la Fédération internationale de laiterie*.

Thème 2003: Rôle de l'alimentation dans la prévention de l'obésité

Dernier délai de remise des travaux: le 31 décembre 2002

Les auteurs ayant écrit des ouvrages scientifiques sur les thèmes proposés sont invités à envoyer un dossier comprenant les pièces suivantes en trois exemplaires:

- curriculum vitae;

- liste des travaux;

- liste des publications;

- tirés à part des cinq plus importants travaux consacrés au thème du prix et publiés au cours des cinq dernières années.

Ces documents doivent être rédigés en langue allemande, française ou anglaise et adressés au président du jury: Professeur Eric Jéquier, Faculté de médecine, Institut de Physiologie, Rue du Bugnon 7, 1005 Lausanne.

* Les pays membres sont:

Afrique du Sud, Albanie, Allemagne,

Australie, Autriche, Belgique, Canada,

Danemark, Espagne, Estonie, Etats-Unis

d'Amérique, Fédération Russe, Finlande,

France, Grèce, Hongrie, Inde, Iran, Irlande, Islande, Israël, Italie, Japon, Kenya,

Koweit, Lettonie, Lithuanie, Luxembourg, Norvège, Nouvelle-Zélande, Pakistan,

Pays-Bas, Pologne, République de Tchéquie, Royaume-Uni, Suède, Suisse, Zimbabwe.

\section{International Award for Modern Nutrition 2003}

The Association of Swiss Milk Producers SMP, Weststrasse 10, CH-3000 Bern 6, invites entries for the 2003 round of its International Award for Modern Nutrition. The prize of CHF 25 000.- will be awarded to a scientist from one of the member states of the International Dairy Federation*.

The subject for 2003: Role of nutrition in the prevention of obesity

Final date for submission: December 31, 2002

Male and female authors of scientific research in the selected fields are invited to submit the following documentation in triplicate:

- Curriculum vitae;

- Research record;

- List of publications;

- Reprints of the five most important studies published in recent years in the selected subject area.

The above mentioned documents must be submitted in German, French or English, and should be addressed to the President of the Jury: Professor Eric Jéquier, MD, Faculty of Medicine, Institute of Physiology, Rue du Bugnon 7, 1005 Lausanne.

* The International Dairy Federation member states are as follows:

Albania, Australia, Austria, Belgium, Canada, Czech Republic, Denmark, Estonia, Finland, France, Germany, Greece, Hungary, Iceland, India, Iran, Ireland, Israel, Italy, Japan, Kenya, Kuwait, Latvia, Lithuania, Luxemburg, Netherlands, New Zealand, Norway, Pakistan, Poland, Russian Federation, South Africa, Spain, Sweden, Switzerland, United Kingdom, USA, Zimbabwe. 


\section{HAE-Vereinigung}

Am 6. September 2001 wurde die Schweizer Vereinigung für Hereditäres Angioedem (HAEVereinigung) gegründet. Diese Patientenvereinigung bietet sowohl HAE-Betroffenen, deren Familienangehörigen, Freunden und Bekannten als auch Ärzten und Pflegepersonal eine Plattform für Informations- und Erfahrungsaustausch. Im benachbarten Ausland gibt es seit längerer Zeit entsprechende Vereinigungen.

Die HAE-Vereinigung sucht den intensiven Kontakt mit spezialisierten Ärzten. In der Folge wurde deshalb im Sommer 2002 die HAE-Expertengruppe gegründet, welche sich aus ärztlich-medizinischer Sicht um diese Krankheit und die davon Betroffenen kümmert. Interessierte Kolleginnen und Kollegen, die gerne in dieser Expertengruppe mitarbeiten möchten, sind eingeladen, mit einem der folgenden Ansprechpartner Kontakt aufzunehmen.

\section{Ärzte der HAE-Expertengruppe}

- Dr. med. C. Bucher

Allergiestation Dermatologie

Universitätsspital Zürich

Tel. 0125511 11;

- PD Dr. med. T. Kühne

LA Universitätskinderspital Basel

Tel. 06168565 65;

- PD Dr. Dr. med. W. A. Wuillemin

LA Hämatologie, Kantonsspital Luzern

Tel. 0412055147.

\section{Kontaktadresse Patientenvereinigung}

Schweizer HAE-Vereinigung

c/o Paula Hunkeler

Jegerlehnerweg 1, 6010 Kriens

Tel./Fax 0413100413.

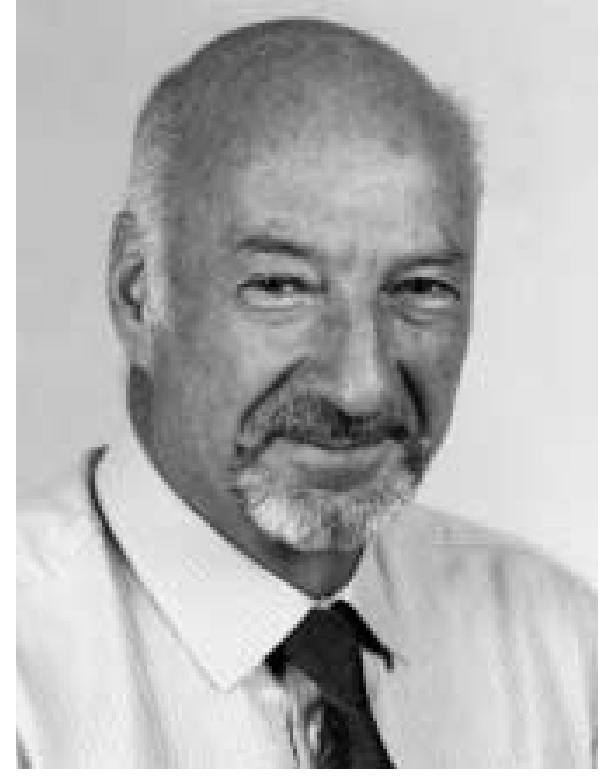

\section{In memoriam}

\section{Prof. Dr. med. Bernhard Georg Weber} (1927-2002)

Am 22. August 2002, kurz nach seinem 75. Geburtstag, verstarb überraschend der ehemalige St. Galler Chefarzt für Orthopädische Chirurgie, Prof. B. G. Weber. Die Schweizer Medizin und internationale Fachwelt verliert mit ihm einen ihrer profiliertesten Vertreter.

Am 7. August 1927 in Basel geboren, absolvierte Weber Schule und Studium in seiner Geburtsstadt. Nach dem 1953 abgelegten Staatsexamen erwarb er sich in Landpraxen, als Hochseeschiffsarzt und in mehreren Schweizer Spitälern medizinische und chirurgische Erfahrungen. Bis zum Aufenthalt an der Orthopädischen Klinik Balgrist war Weber dennoch nie sicher, ob er nicht doch Architekt statt Arzt hätte werden sollen. Überzeugt, den richtigen Beruf gewählt zu haben, wurde er erst am Balgrist, als er erkannte, dass die in der Orthopädie vollzogene Synthese von Medizin, Technik und Handwerk seinen Neigungen am besten entspricht. Nach Abschluss der Orthopädischen Fachausbildung an der Klinik Balgrist vervollständigte Weber am Spital Uster die Ausbildung zum Allgemeinchirurgen.

Webers St. Galler Biographie beginnt mit der wegweisenden Entscheidung der St. Galler Regierung, die grosse Klinik für Chirurgie in eine Klinik für Allgemeinchirurgie und eine Orthopädische Klinik aufzuteilen und der Orthopädischen Klinik den Auftrag zu erteilen, Unfallchirurgie des Bewegungsapparats und klassische Orthopädie unter einem Dach zu praktizieren. Das für die Schweiz zur damaligen Zeit neue Konzept wurde bald Vorbild für andere Schweizer Spitäler. Weitblick bewies auch die Wahl von Prof. M. E. Müller zum Chefarzt der im November 1960 eröffneten neuen Klinik. St. Gallen wurde damit zur ersten grossen klinischen Plattform der noch jungen AO, der Arbeitsgemeinschaft für Osteosynthesefragen.

Müller und Weber hatten sich an der Klinik Balgrist kennengelernt. Als er von Müller gefragt wurde, ob er am Aufbau der neuen Klinik mitarbeiten wolle, hat Weber die Herausforderung sofort angenommen, obwohl er bereits einen Vertrag für eine attraktive Stelle bei Prof. Stinchfield in New York in der Tasche hatte und sich mit seiner Frau Alice schon in England befand. Weber war zunächst sechseinhalb Jahre Oberarzt, bis Müller 1967 St. Gallen verliess und er als dessen Nachfolger gewählt wurde. 
Ein eindrucksvoller klinischer und wissenschaftlicher Leistungsausweis kennzeichnet Webers Tätigkeit an der St. Galler Klinik. Aus seinen Vorträgen, Einzelpublikationen und Büchern sowie aus der Arbeit in Kommissionen der AO kamen wichtige Beiträge zur modernen Orthopädischen Chirurgie. Originalität, klare Linien und das stetige Streben nach einer kohärenten logischen Systematik prägen Webers Publikationen. 1967 honorierte die Universität Bern seine Lehrtätigkeit und wissenschaftliche Arbeit mit der venia legendi und 1974 mit der Honorarprofessur.

Herkömmliches kritisch in Frage zu stellen und neue Ideen zu generieren war eine von Webers Stärken. Schon zu Beginn der sechziger Jahre setzte er sich für die operative Stabilisierung der Wirbelsäule bei Querschnittlähmungen ein. Eine von Weber ebenfalls in den sechziger Jahren veröffentlichten Monographie mit einer biomechanisch fundierten neuen Klassifizierung der Sprunggelenksverletzungen hat die Behandlung dieser Verletzungen entscheidend beeinflusst. Später veröffentlichte er unter anderem Beiträge zur Technik der Zuggurtungsosteosynthese, Bücher zur Behandlung von Pseudarthrosen, zu Verletzungen im Kindesalter, zum Fixateur externe und über seltene Osteosynthesen. Nicht unerwähnt soll bleiben, dass Weber schon sehr früh die Bedeutung der biologischen Osteosynthese erkannte und in diesem Zusammenhang immer wieder darauf hinwies, dass Pseudarthrosen heilbar sind, toter Knochen aber nie zusammenwächst.

Weber entwickelte ferner das erste modulare künstliche Hüftgelenk und befasste sich zeitlebens mit dessen Verbesserung. Es charakterisiert seinen Perfektionismus und sein handwerkliches Geschick, dass er die Modelle für die Kunstgelenke jeweils selbst anfertigte und so lange an Leichenknochen erprobte, bis er mit dem Resultat zufrieden war. Besonders hervorzuheben ist Webers jahrelanger Einsatz für die Reinraumtechnik zur Senkung der damals noch recht markant gewesenen postoperativen Infektionsrate beim künstlichen Gelenkersatz. Breite Wirkung erzielte er auch mit dem «Minimaxprinzip». Es besagt, dass der angestrebte Operationserfolg immer mit möglichst einfachen Mitteln, d.h. mit dem geringsten iatrogenen Trauma, erzielt werden soll. Mit den jetzt allgegenwärtigen Bestrebungen um die Minimalisierung des chirurgischen Traumas hat das Prinzip wieder besondere Aktualität erlangt.

Auf dem von M. E. Müller gelegten Fundament hat Weber massgeblich zum hohen Ansehen der Klinik beigetragen. Sein Ruf als begnadeter Chirurg und Wissenschaftler wirkte bald weit über die Landesgrenzen hinaus. Aus dem Inund Ausland kamen Patienten sowie Ärzte, die unter Webers Obhut das orthopädische Handwerk erlernen oder sich als Gastärzte weiterbilden wollten. Weber war ein talentierter Lehrer, der sein Wissen faszinierend anschaulich weitergeben konnte. Sein subtiler Basler Humor liess bei den täglichen Rapporten nie Langeweile aufkommen. Man schätzte seinen kritischen Intellekt und seine Fähigkeit, das Wesen eines Problems rasch zu erkennen und es präzise, meist auch originell formuliert, darzulegen. Weber tolerierte fremde Meinungen, war zugänglich für Ideen, konnte aber dennoch geradlinig seinen Überzeugungen treu bleiben. Humor, gemeinsam betriebener Sport und gesellige Anlässe betrachtete Weber für den Klinikbetrieb förderlich und ebenso nötig wie gute ärztliche Arbeit. Für seine Mitarbeiter hat er sich auch nach deren Abgang von der Klinik immer wieder eingesetzt. Nicht wenige verdanken ihre Karriere seiner Fürsprache.
Den Beruf betrachtete Weber als sein Hobby. Der totale Einsatz für das, was er als seine eigentliche Aufgabe ansah, für die Patienten zu sorgen und am medizinischen Fortschritt mitzuwirken, erforderte jedoch von seinem gesellschaftlichen und privaten Leben erheblichen Tribut und wäre ohne verständnisvolle Unterstützung durch die Familie nicht möglich gewesen. Die spärliche Freizeit war dann auch ganz der Familie, der modernen Kunst und dem Sport gewidmet, insbesondere dem Skilaufen.

Der Verzicht auf zwei Berufungen an Universitätskliniken lässt erahnen, wie sehr sich Weber mit seiner Klinik identifizierte. Dass er sich dennoch Ende 1986 entschlossen hat, sein Amt niederzulegen, um an der Orthopädie am Rosenberg privat weiterzuarbeiten, kam für viele überraschend. Dafür massgebend war wohl, dass Weber sich in erster Linie als an der Front tätiger Arzt und Wissenschaftler sah und die rasch anwachsende Beanspruchung durch viele andere Aufgaben als störende Behinderung empfand. Er wollte sich wieder mehr den Patienten, wissenschaftlichen Projekten und nicht zuletzt auch der Familie widmen können. An der Orthopädie am Rosenberg hatte für Weber ein neuer Lebensabschnitt begonnen, dessen Vorzüge er durchaus zu schätzen wusste. Dennoch blieb er immer seiner alten Klinik zugeneigt und verfolgte hochinteressiert die Entwicklungen im fachlichen Bereich. Ein Herzversagen hat ihn nun mitten aus einer nahezu ungebrochenen ärztlichen und wissenschaftlichen Aktivität herausgerissen. Mit seiner Familie trauern viele Freunde, Kollegen, dankbare Schüler und Patienten.

F. Magerl, St. Gallen 